\title{
Avances en el conocimiento de la dinámica forestal: el papel del monitoreo regional
}

\author{
Gray, A.N. \\ USDA Forest Service, Pacific Northwest Research Station \\ 3200 SW Jefferson Way \\ Corvallis, OR 97331 EEUU
}

*Autor para correspondencia: agray01@fs.fed.us

\section{Resumen}

Los inventarios forestales nacionales proporcionan datos básicos sobre la biodiversidad, la integridad de los ecosistemas, y la productividad de los recursos naturales en países de todo el mundo. Con mediciones repetidas, estos datos son una base de información muy poderosa para avanzar en nuestro conocimiento de los procesos ecológicos y su respuesta a la gestión forestal y las perturbaciones naturales. Aunque el análisis de datos dispares ("big data") con nuevas técnicas (por ejemplo, aprendizaje automático) puede ser de utilidad, no hay sustituto para un diseño estadístico riguroso y mediciones cuidadosas para hacer avanzar la ciencia forestal. Con una muestra aleatoria que cubre regiones enteras y el seguimiento de parcelas permanentes se puede caracterizar la dinámica de comunidades vegetales tal y como ocurren en el paisaje, en vez de confiar en estudios de sitios controlados y seleccionados subjetivamente. Con una mejor accesibilidad a los datos de los inventarios se está llegando a conclusiones que contradicen resultados y conceptos aceptados en la literatura científica. En esta presentación, revisamos conceptos básicos de la ciencia del monitoreo forestal, y damos ejemplos de resultados sobre los efectos de los incendios forestales, el impacto del declive de especies forestales y el flujo de carbono. Finalmente, discutiremos limitaciones, oportunidades y problemas actuales de la ciencia de monitoreo.

Palabras clave: cambio ambiental, diseño experimental, incendio, inventario. 


\section{Introducción}

Los inventarios forestales nacionales (IFN) proporcionan información esencial para el desarrollo de recursos naturales en muchos países del mundo. Los IFN también son importantes para documentar la sostenibilidad de la gestión forestal y el bienestar del medio ambiente. En ellos es normal obtener información sobre área de bosque, su productividad, volumen maderable, y sus cambios con el tiempo. Además, con los datos de IFN se puede hacer inferencia sobre la biodiversidad, hábitat de fauna, y la integridad de los ecosistemas. Esta información es útil para la realización de planes nacionales y para evaluar el cumplimiento de acuerdos internacionales. La repetición de mediciones de IFN, es decir el monitoreo, forman una base de información poderosa para avanzar el conocimiento de procesos ecológicos y la respuesta de los ecosistemas a la gestión forestal y las perturbaciones naturales.

La realización de inventarios y el monitoreo a veces tiene la reputación en la comunidad científica de ser simple y de menor interés. Muchos tienen la impresión que en el inventario solo se trata de medir cosas simples, lo cual es bastante fácil y sus resultados no tienen mucha importancia. Al contrario, queremos demostrar que los inventarios pueden ser la mejor forma para contestar preguntas críticas sobre el manejo sostenible de nuestros bosques. En este artículo, queremos discutir:

a) Los objetivos y diseños de inventarios forestales.

b) La función de parcelas en la estimación: inferencia basada en el diseño o en un modelo.

c) Los retos de la remedición: estimando cambio con confianza.

d) Ejemplos de varios usos de inventarios.

\section{Objetivos y mediciones de inventarios}

Los IFN en muchos países han operado desde hace muchos años (p.ej., desde 1928 en los Estados Unidos). Tradicionalmente el objetivo primario fue estimar la cantidad y calidad de volumen maderable y la sostenibilidad de la gestión de los bosques en cada país. Aunque todavía de primaria importancia, recientemente se han incorporado otros objetivos en muchos de los IFN. Ejemplos de preguntas que los IFN están diseñados para contestar incluyen:

- ¿Cuál es la superficie de bosque, por tipo de comunidad, manejo, protección, y riesgo de incendio?

- ¿Cuál es la cantidad y calidad de volumen, biomasa, o carbón?

- ¿Cuál es la calidad de hábitat y la función hidrológica que dan los bosques?

- ¿Cuánto está cambiando cada parámetro de interés?

- ¿Cuáles son las causas primarias que explican los cambios (manejo, perturbaciones naturales, cambio climático)? 
Un ejemplo del uso de IFN y otros datos para contestar diversas preguntas es el Proceso de Montreal de 1994 que desarrolló criterios e indicadores para la conservación y gestión sostenible de los bosques templados y boreales. Doce países son miembros del acuerdo y representan el $90 \%$ de los bosques templados y boreales. Los criterios e indicadores incluyen: biodiversidad, capacidad productiva, suelos y agua, el ciclo de carbono, y el beneficio social a largo tiempo. Los datos de IFN informan los criterios, o directamente (p.ej., cambios en área de bosque) o con interpretación de modelos (p.ej., cambios en área de hábitat de especies en peligro de extinción).

Los IFN son diseñados para responder preguntas a nivel estratégico; en términos prácticos, áreas o tipos de vegetación muestreadas por 30 o más parcelas (en la IFN de los Estados Unidos, 30 parcelas representan 72000 ha). Pero los miles de parcelas y millones de medidas también son útiles para una gran variedad de preguntas, y, en combinación con la teledetección, para hacer mapas (modelos) útiles para planear la gestión, incluso de rodales individuales.

Las mediciones fundamentales de todos los IFN son de árboles vivos y el uso de la tierra. Típicamente el área de cada parcela está dividido por uso, por ejemplo bosque, vegetación natural sin bosque, agricultura, urbanización, y agua. Mediciones de individuo de árboles vivos incluyen: especie, diámetro, altura, condición de copa, tipo y causa de daños, crecimiento, mortalidad, extracción de madera, y crecimiento histórico. Muchos IFN también evalúan el tamaño y nivel de descomposición de madera muerta, con mediciones de árboles muertos en pie, y de madera caída. Es común también estimar la cobertura de matorral, por tipo o especie, incluyendo la búsqueda de plantas invasoras. En muchos casos los IFN también evalúan suelos y hojarasca, con mediciones de erosión en el campo, y de densidad, carbono, y nutrientes en el laboratorio. Los datos de la IFN de los Estados Unidos que están disponible en la red están documentados en Woudenberg et al. (2010). La muestra de parcelas del IFN también es útil para mediciones de fauna con trampas para insectos, observaciones de pájaros, o con cámaras remotas (p. ej., Morton et al. 2009).

Cada IFN tiene que hacer compromisos sobre las mediciones más importantes, y estar listo para responder las preguntas críticas cotidianas. Mediciones que necesitan mucha pericia y conocimiento pueden complicar el diseño y el coste del inventario. Pero hay opciones para integrar distintas prioridades, por ejemplo tomar algunas de las medidas en una submuestra. Por ejemplo, para medir la comunidad de sotobosque, se pueden hacer medidas simples de la cobertura de especies dominantes realizadas por personal regular, o medidas detalladas de todas especies hechas por botánicos, y solo en tiempos de fenología ideal (Schulz et al. 2009).

\section{Diseño experimental y la función de parcelas}

Cada experimento o estudio científico necesita empezar con definiciones específicas y el reconocimiento de los supuestos hechos para el estudio. Definiciones importantes incluyen el alcance de la investigación y los resultados, y los objetos de 
estudio (y por sustracción, los que no están incluidos). Los supuestos pueden ser más difíciles de reconocer. Por ejemplo, si un aficionado de futbol lee el título “¡USA Campeones mundiales de futbol!” sería muy difícil de creer, ya que la copa mundial de mayor atención nunca ha sido ganado por los Estados Unidos. Pero el título es verdad, porque sí ha pasado que el equipo nacional de los Estados Unidos ha ganado la copa mundial femenina de futbol tres veces desde el año 1990.

El diseño experimental es crítico para la ciencia. En la enseñanza de la ciencia y estadística, típicamente el énfasis es en experimentos diseñados para probar una hipótesis. El diseño típico de muchos estudios consiste en centrarse en una pregunta, un proceso, y muchas veces un tipo de bosque o comunidad vegetal. Los aspectos clave del diseño son la aleatoriedad de asignación de sitios a tratamientos y la repetición de tratamientos. Las investigaciones típicamente imponen tratamientos específicos, por ejemplo métodos de selvicultura. Para entender la pregunta clave se minimizan otros aspectos o procesos que pueden afectar el resultado. Casi siempre el alcance del tema es limitado a unos tratamientos seleccionados, y el alcance geográfico es limitado porque la repetición es cara. Aunque a veces las mediciones en experimentos pueden ser simples y similares a los de IFN, muchas veces las mediciones son más detalladas y frecuentes, y enfocados en procesos ecológicos además de cambios en la estructura vegetal.

El diseño experimental también es crítico para el seguimiento hecho en los IFN. La población estadística es todos los bosques en un área determinada (p.ej., un país). El diseño es una muestra de la población con parcelas con probabilidades de inclusión conocidas; evitar el sesgo tiene una importancia primordial. No se intenta controlar la variación entre bosques, al contrario se intenta medir la variación en la proporción en que ocurre. Las definiciones claras y manejables tienen una importancia fundamental porque, por ejemplo, es necesario dividir toda la tierra del país entre bosque y no bosque, y todas las especies y formas de plantas en el país entre árbol y no árbol. Aunque el énfasis de muchos IFN son en el diseño y la medición en campo, la fotogrametría ayuda a navegar y a clasificar parcelas de campo, y la teledetección es integral en la estimación (p.ej., Dunham et al. 2002).

A causa de las diferencias en objetos y diseños, las características de las parcelas medidas en experimentos suelen ser bastante diferentes a las de los inventarios. Cuando hacemos investigaciones en ecología o selvicultura, es típico buscar bosques homogéneos que respondan a las preguntas específicas de interés, y que tienen la tendencia a ser densos, con árboles grandes, o sin perturbaciones recientes (Fig. 1). En contraste, muchos bosques muestreados por inventario son bastante variables, con condiciones mixtas derivadas de la acción de incendios, topografía, o proximidad a ríos o prados (Fig. 2). Estas condiciones y variaciones típicamente son evitadas en las investigaciones forestales, excluyendo de hecho una gran proporción de los bosques reales. El problema es cuando investigadores usan sus datos y modelos para estimar a gran escala sin considerar las varianzas que no midieron. Por ejemplo, hay artículos científicos (p.ej., Zheng et al. 2011; Krankina et al. 2012) que estiman que los bosques en el noroeste de los EEUU están secuestrando el doble de carbono de los que estimamos con el inventario (Gray y Whittier, 2014). Sin duda, las investigaciones tí- 
picas que seleccionan bosques subjetivamente pueden tener gran valor para revelar procesos ecológicos en gran detalle, pero es importante considerar cómo los resultados se aplican al bosque en general.

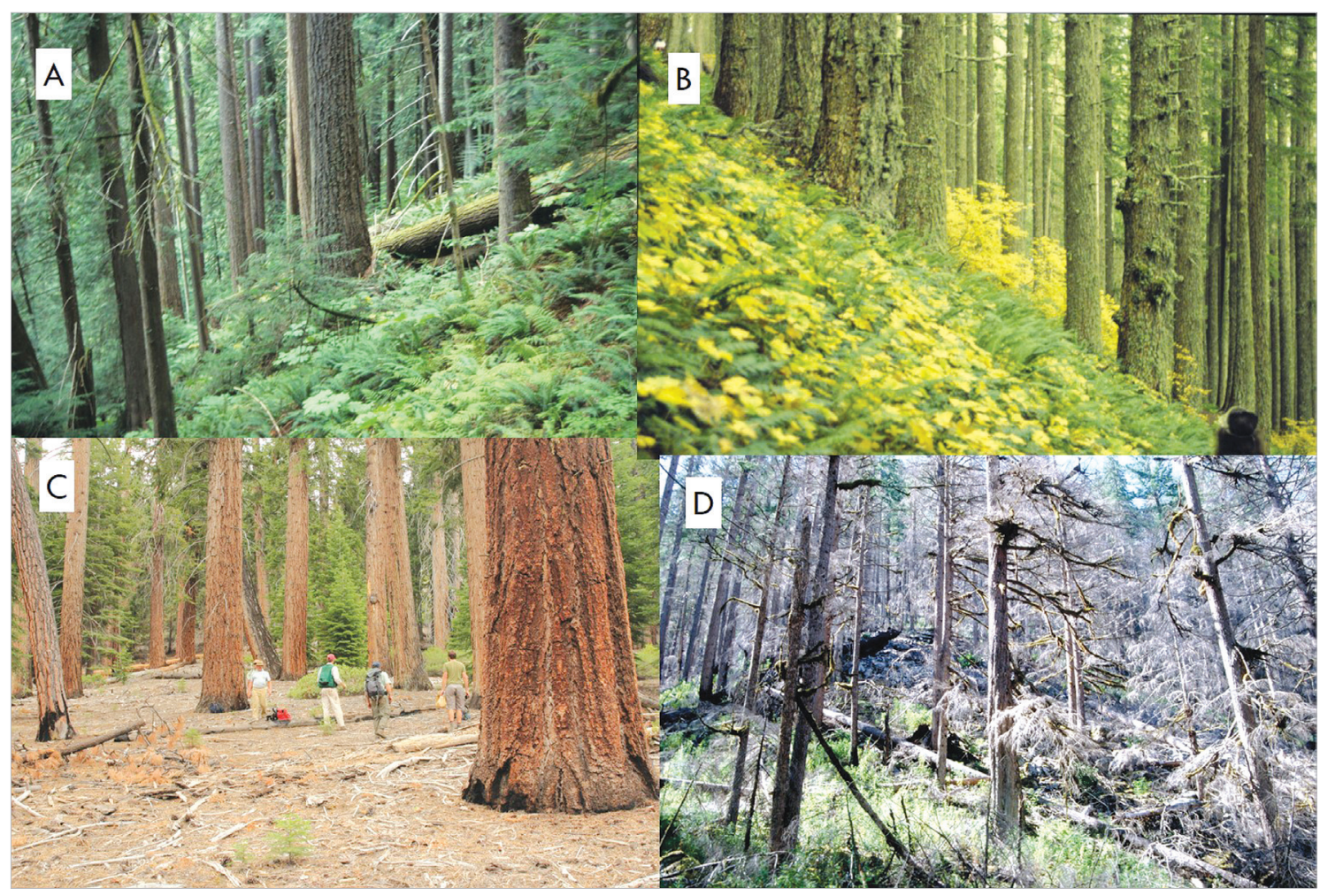

Figura 1. Las investigaciones en ecología o selvicultura frecuentemente se hacen en bosques homogéneos que conforman a las preguntas específicas de interés. Estos ejemplos son bosques de tipo: A) PseudotsugaTsuga, Oregon, B) Pseudotsuga, Oregon, C) Abies magnifica, California, D) Incendio reciente en Pseudotsuga-Tsuga, Washington.

En el análisis de inventarios, tenemos mediciones directas y detalladas, distribuidas en una muestra con probabilidad conocida. No hace falta hacer ningún supuesto; los cálculos se hacen directamente de las parcelas. La varianza está bien definida por las mediciones de la parcela y la post-estratificación con información auxiliar como teledetección o clima (Scott et al. 2005). Por el contrario, hay expertos que nos han dicho que solo tienen que instalar parcelas seleccionadas en diferentes condiciones y en zonas de fácil acceso para hacer un modelo usando teledetección y estimar el parámetro de interés (p.ej., volumen de madera), con muy poco gasto. Pero hacer ese tipo de estimación requiere el supuesto de que las parcelas seleccionadas han muestreado la variación de condiciones actuales y que el modelo es correcto. Para calcular cambio de condiciones (p.ej. el flujo de carbono), hay que comparar resultados de dos modelos, posiblemente con cambios en la tecnología de teledetección. ¿Y qué confianza vamos a tener en los cálculos si ha habido incendios grandes pero ninguna de las parcelas subjetivas están en una zona de incendio? 


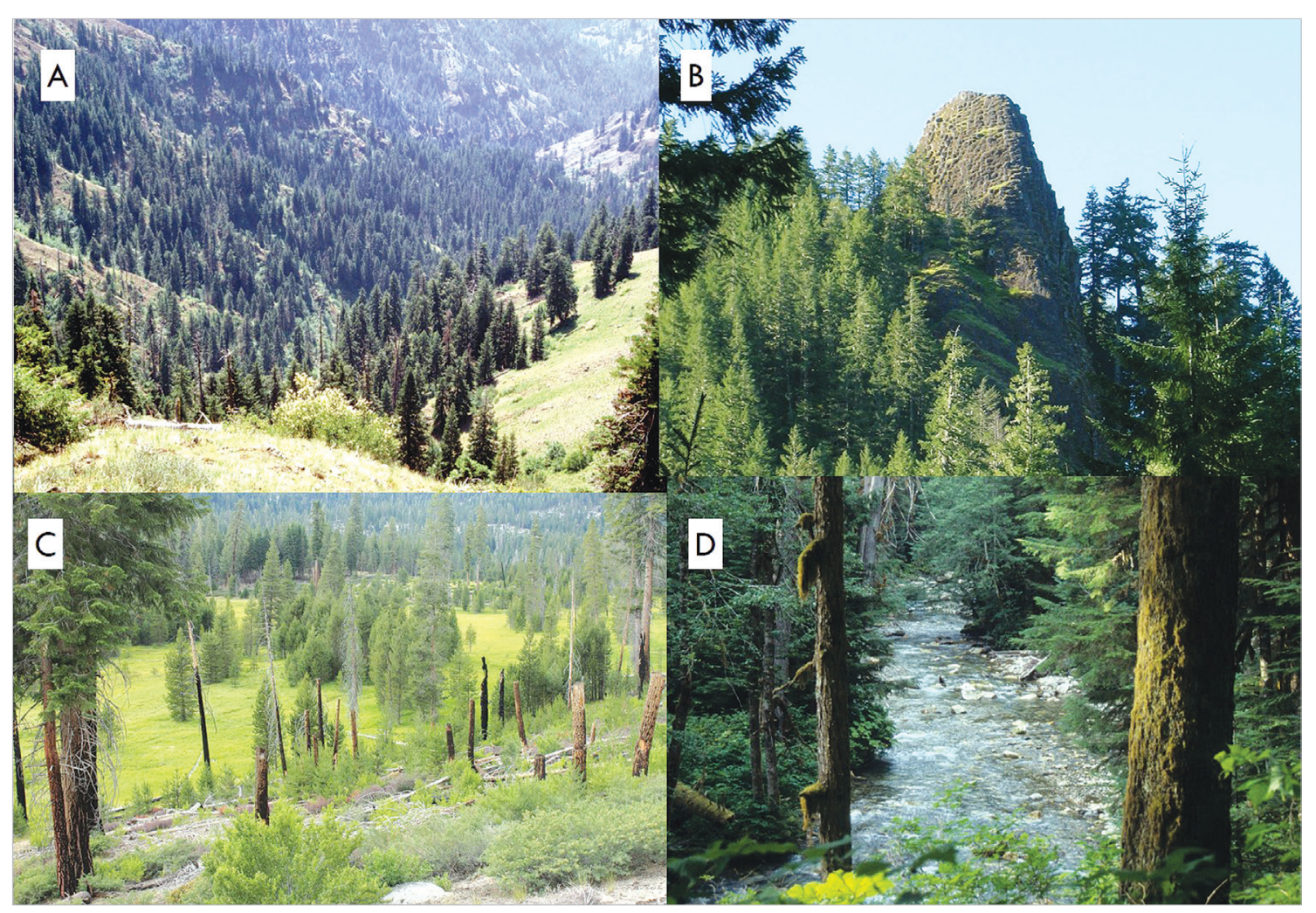

Figura 2. Las parcelas en inventarios frecuentemente caen en bosques no homogéneos, por ejemplo: A) Prados situados junto a bosques densos. B) Áreas de roca entre bosque. C) Condiciones mixtas originadas por varios incendios. D) Áreas de ríos entre los bosques.

El papel de la parcela depende en si el análisis está basada en diseño o en modelo. El uso de imágenes de satélite clasificadas para la post-estratificación de inventarios es típico (p.ej., Westfall et al. 2011). En este caso la estimación está basada directamente en las parcelas, y las imágenes de satélite sirven para reducir la varianza de los cálculos. En otro caso, las mismas parcelas de inventario, y datos de otras parcelas si existen, se pueden usar en un modelo con imágenes de satélite (p.ej., de volumen total) para construir mapas de la región de interés (Ohmann y Gregory 2002). La ley en los Estados Unidos requiere que los datos del IFN estén calculados sean accesibles al público (https://www.fia.fs.fed.us/tools-data/index.php) en un periodo máximo de 6 meses después de ser medidos. Esto ha resultado en la utilización de los datos por investigadores, gestores en varias agencias, empresas forestales y el público. El IFN ha hecho varias herramientas para facilitar el acceso y utilización de los datos. Al fin del día, ser útil es la razón para tener un inventario, y cuanta más gente utilice el inventario mejor podremos contribuir a la información que necesita el público.

\section{Los retos de remedición}

El seguimiento, que depende en gran parte en la remedición de parcelas, no es 
igual al inventario, que es basado en una única medición. En la estimación con inventarios, intentamos estimar valores grandes (p.ej., área en encinas grandes o volumen maderable en la provincia de Cáceres), que muchas veces tienen distribuciones y valores no muy variables. Pero en el seguimiento, el objetivo es estimar cambios (p.ej., superficie de deforestación, cambio de uso, crecimiento y mortalidad de robles en la provincia de Cáceres). Las cantidades de los cambios típicamente son más pequeñas y mucho más variables que los totales en un punto determinado. Por ejemplo los bosques nacionales de Oregón y Washington en el noroeste de los Estados Unidos se pueden agrupar en 5 regiones distintas (Fig. 3). En un análisis de flujo de carbono en estos

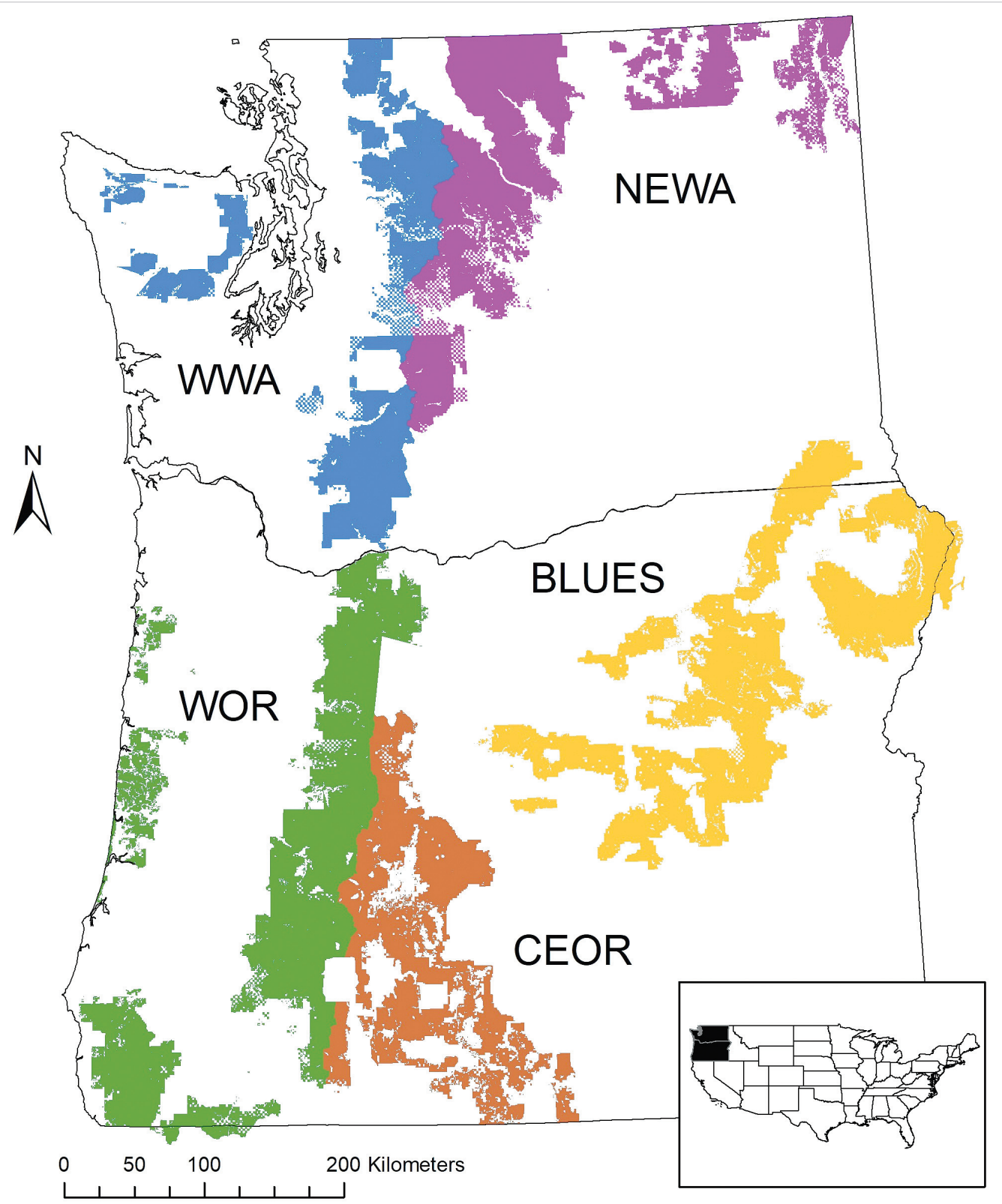

Figura 3. Mapa de cinco regiones distintas en los bosques nacionales del noroeste de los Estados Unidos. 
bosques (Gray y Whittier 2014), fue claro que la varianza del flujo de carbono (calculado como el porcentaje del promedio en error estándar) es en muchos casos diez veces más grande que la varianza de carbono total o área de bosque (Tab. 1). La precisión de estimaciones de cambio basadas en remedición típicamente es más pequeño que la precisión de cantidades solo basadas en una medición. Estimar valores de cambio con más precisión requiere análisis basados en más parcelas, por ejemplo extendiendo el área de interés o los tipos de bosques incluidos, o aumentado el número de parcelas en una región de interés.

Tabla 1. Comparación de valores y errores en las etsimaciones de área, carbono total, y variación de carbono anual en los bosques nacionales en Oregon y Washington, EEUU. SE = error estándar, $\mathrm{SE} \%=$ porcentage error estándar.

\begin{tabular}{|c|c|c|c|c|c|c|c|c|c|}
\hline \multirow[b]{2}{*}{ Estudio } & \multicolumn{3}{|c|}{ Area de bosque (ha) } & \multicolumn{3}{|c|}{ Carbono total $(\mathrm{Mg} / \mathrm{ha})$} & \multicolumn{3}{|c|}{$\begin{array}{c}\text { Cambio de carbono annua } \\
(\mathrm{Mg} / \mathrm{ha} / \mathrm{an})\end{array}$} \\
\hline & Total & SE & $\mathrm{SE} \%$ & Promedio & SE & SE\% & Promedio & SE & SE\% \\
\hline BLUES & $2,103,034$ & 17,177 & $0.8 \%$ & 61.2 & 0.9 & $1.5 \%$ & 0.26 & 0.03 & $13.1 \%$ \\
\hline CEOR & $1,450,669$ & 8,125 & $0.6 \%$ & 60.4 & 1.2 & $2.0 \%$ & 0.40 & 0.08 & $20.4 \%$ \\
\hline NEWA & $1,780,247$ & 20,548 & $1.2 \%$ & 88.4 & 1.9 & $2.2 \%$ & 0.77 & 0.07 & $8.6 \%$ \\
\hline WOR & $2,388,646$ & 9,197 & $0.4 \%$ & 177.2 & 2.4 & $1.4 \%$ & 0.87 & 0.10 & $11.2 \%$ \\
\hline WWA & $1,354,126$ & 13,036 & $1.0 \%$ & 182.3 & 3.3 & $1.8 \%$ & 0.97 & 0.17 & $17.3 \%$ \\
\hline Total & $9,076,723$ & 31,155 & $0.3 \%$ & 115.0 & 0.9 & $0.8 \%$ & 0.65 & 0.04 & $6.3 \%$ \\
\hline
\end{tabular}

El reto del seguimiento en los IFN es hacer estimaciones fiables de cambio mientras otras variables que afectan el inventario están cambiando también (Fig. 4). En este ejemplo las variables fundamentales que queremos estimar y entender son el crecimiento, la mortalidad, y producción de los arboles vivos en una región. Esos cambios están afectados por varios agentes de cambio que queremos entender, incluso el incendio, construcción de caminos, el movimiento de ríos (que pueden quitar o añadir bosque). Pero también hay cambios que complican el seguimiento. Es típico que el presupuesto fluctúa con la economía y la política del país. Esto fuerza cambios en diseño y mediciones, que complican el cálculo de cambios con remedición. Los procesos de entrenamiento y control de calidad tienen que mantener la constancia de las mediciones cuando el personal cambia con el paso del tiempo. Siempre hay nuevas técnicas, o mejores ecuaciones, que van a cambiar los cálculos, y fuerzan el recalcular los valores previos para poder compararlos. El peor cambio de todos es el cambio de definiciones, porque el cambio puede afectar a la probabilidad de muestreo de la población de interés. Por ejemplo, en los Estados Unidos hemos ajustado la definición de bosque para compatibilizarla con definiciones internacionales. El cálculo de cambios de bosque fiables requiere mediciones extras para comparar el efecto del cambio y ajustar valores previos a la nueva definición (p.ej., Azuma y Gray 2014). 


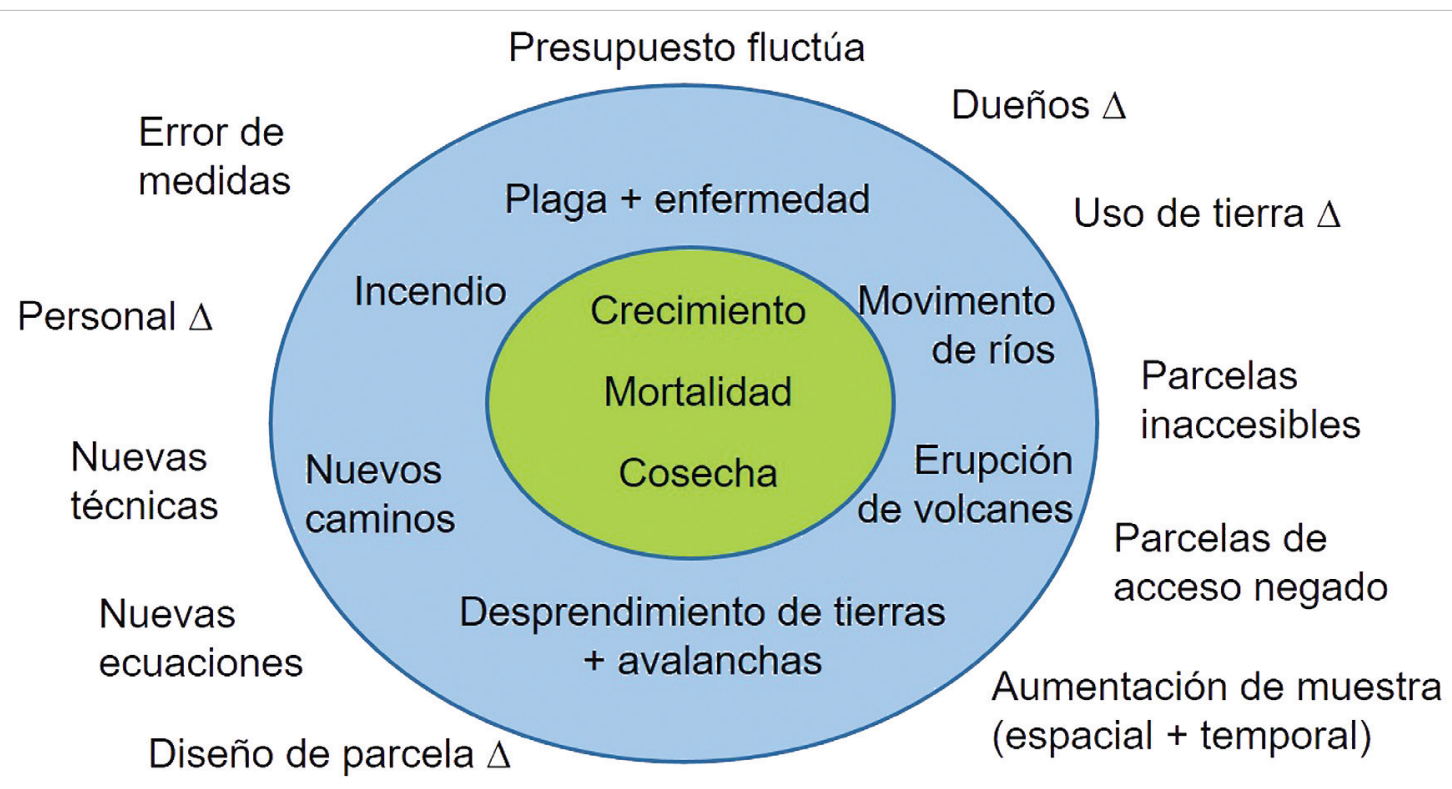

Cambios de definiciones

Figura 4. Gráfico de los cambios primarios que ocurren en bosques y otros hechos que afectan a los inventarios y que complican la estimación del cambio. En el centro, en verde, están los cambios que ocurren a individuos. Alrededor, en azul, están los procesos naturales que resultan en cambios en bosques. Y fuera del círculo azul están los cambios que afectan el conjunto del inventario.

\section{Ejemplos de investigaciones de cambios y sus causas}

Con los datos de seguimiento de los IFN se han realizado una gran variedad de investigaciones, desde descripciones básicas de cambio de área de bosque en diferentes condiciones hasta análisis de la importancia del clima, competición, y topografía en la abundancia de diferentes especies. Tenemos ejemplos de varias investigaciones que hemos hecho en la costa oeste de los Estados Unidos.

Hay mucha discusión sobre el nivel del flujo de carbono en diferentes tipos de bosques y en respuesta a perturbaciones naturales. Hicimos un análisis de seguimiento en bosques nacionales de Oregón y Washington, donde se habían medido cambios en arboles vivos, muertos en pie, y madera caída (Gray y Whittier 2014). En estos bosques públicos en este tiempo no hay mucha corta de madera, y el crecimiento de árboles vivos es mayor que la mortalidad, especialmente en áreas húmedas. El cambio neto en áreas protegidas es menor, porque los bosques son de edad avanzada, y el cambio es negativo (perdiendo carbono) a causa de incendios en bosques secos de áreas protegidas. Pero la mayoría del carbono en árboles que mueren se queda en el ecosistema como madera muerta, donde se descompone lentamente. Es decir, el efecto de incendios donde ocurren en el flujo de carbono, es similar al efecto del aclareo parcial en estos bosques.

En un análisis de la variación de flujo de carbono por tipo de bosque, productividad y edad la variable de mayor importancia fue la productividad del sitio (Gray et 
al. 2016). El crecimiento de árboles vivos subió rápidamente con la edad al máximo a la edad de 40 años y se quedo a ese nivel, bajando hasta el $\sim 70 \%$ en los sitios más productivos. Con el aumento de la densidad con la edad, la mortalidad aumenta también, hasta que después de los 200 años el cambio neto de árboles vivos se queda cerca de cero. El carbono en madera muerta, continua aumentando ligeramente con la edad, con el resultado de que en los bosques de mayor edad (>200 años) todavía hay aumento de carbono amenazado $(0.15-0.70 \mathrm{Mg} \mathrm{C} / \mathrm{ha} / \mathrm{yr})$.

Otro estudio usó mediciones especiales que se hicieron el año después de que ocurriesen incendios (Eskelson et al. 2016). Los resultados indican que los bosques no se comportan con la caricatura común del efecto de incendios, donde todos los arboles vivos mueren y se convierten en madera muerta que se descompone por tiempo. Al contrario, la mayoría de parcelas (79\%) no se quemaron con alta intensidad. Además, los bosques con mayor contenido de carbono tienden a arder con menos severidad. Aunque existía pérdida de carbono por descomposición en los primeros seis años después de incendios de alta severidad, el efecto fue pequeño y no fue estadísticamente significativo.

Un buen ejemplo de la combinación de parcelas de inventario con información geográfica y modelos de selvicultura es el proyecto BioSum (Fried et al. 2017). El análisis usa una red de carreteras y la ubicación de parcelas para estimar los costes de saca a plantas de energía potenciales, y el costo o las ganancias de usar diferentes tipos de claras y tratamientos para reducir el riesgo de incendios. Los resultados pueden informar la probabilidad de suceso de diferentes tipos de manejo, y el potencial económico de esas actividades. En un análisis de opciones para tratamientos en los montes Blue de Oregón, el riesgo de incendio de alta severidad podría ser reducido efectivamente en el $35 \%$ de los rodales, y el efecto perdurará 40 años después (Loreno et al. 2015).

Los Estados Unidos todavía no tienen inventario en 15\% de su bosque-40 millones de hectáreas en el interior de Alaska, que es muy caro de medir. Aunque el valor de la madera es poco, el potencial de emitir gran cantidad de carbono con el cambio climático existe. Hemos hecho un estudio piloto que usa parcelas distribuidas a 1/5 de la densidad normal, y usa transectos de Lidar y sensores hiperespectrales para hacer la post-estratificación (Andersen et al. 2015). Al demostrar que podíamos instalar parcelas y utilizar esta nueve tecnología de teledetección, en 2016 hemos empezado a hacer el inventario del interior de Alaska.

\section{Conclusiones}

Al finalizar, unos puntos clave de mi presentación:

- El inventario es una ciencia que complementa bien las investigaciones tradicionales ecológicas y silvícolas.

- El seguimiento es más complicado que el inventario, pero la ganancia en comprensión es grande.

- Lidar y teledetección aumentan y extienden la información de parcelas, pero no la reemplaza. 
- Los datos abiertos mejoran la utilidad de los datos y también las discusiones sobre la gestión basadas en información científica.

\section{Agradecimientos}

Gracias a Vicente Monleon por las discusiones sobre el diseño propio de inventarios; a Vicente, Olalla Diaz Yanez y Francisco Mauro por sus comentarios y ayuda con el vocabulario de la presentación; y al personal de FIA por gran esfuerzo en la colección y procesamiento de los datos de inventarios.

\section{Bibliografía}

Andersen, H.E., Babcock, C., Pattison, R., Cook, B., Morton, D., Finley, A. 2015. The 2014 tanana inventory pilot: A USFS-NASA partnership to leverage advanced remote sensing technologies for forest inventory. In: Stanton, S.M., Christensen, G.A. (Eds). 2015. Pushing boundaries: new directions in inventory techniques and applications: Forest Inventory and Analysis (FIA) symposium 2015. 2015 December 8-10; Portland, Oregon. Gen. Tech. Rep. PNW-GTR-931. Portland, OR: U.S. Department of Agriculture, Forest Service, Pacific Northwest Research Station: 40-41. https://www.fs.usda.gov/treesearch/pubs/50169

Azuma, D.L.; Gray, A.N. 2014. Effects of changing forest land definitions on forest inventory on the West Coast, USA. Environmental Monitoring and Assessment. 186: 1001-1007. http://treesearch.fs.fed.us/pubs/49081

Dunham, P.A., Weyermann, D.L., Azuma, D.L. 2002. A comparison of stratification effectiveness between the National Land Cover Data set and photointerpretation in western Oregon. In: McRoberts, R.E., Reams, G.A., Van Deusen, P.C., Moser, J.W. (Eds.), Proceedings of the Third Annual Forest Inventory and Analysis Symposium, Traverse City, Michigan, October 17-19, 2001. USDA Forest Service North Central Research Station, St. Paul, MN, pp. 63-68. https://www.treesearch.fs.fed.us/pubs/14429

Eskelson, B.N.I.; Monleon, V.J.; Fried, J.S. 2016. A 6 year longitudinal study of post-fire woody carbon dynamics in California's forests. Canadian Journal of Forest Research. 46: 610-620. https://www.fs.usda.gov/treesearch/pubs/53158

Fried, J.S., Potts, L.D., Loreno, S.M., Christensen, G.A., Barbour, R.J. 2017. Inventory-based landscape-scale simulation of management effectiveness and economic feasibility with BioSum. Journal of Forestry.115: 249-257.

Gray, A.N., Whittier, T.R. 2014. Carbon stocks and changes on Pacific Northwest national forests and the role of disturbance, management, and growth. Forest Ecology and Management. 328: 167-178. http://www.treesearch.fs.fed.us/pubs/46566

Gray, A.N., Whittier, T.R., Harmon, M.E. 2016. Carbon stocks and accumulation rates in Pacific Northwest forests: role of stand age, plant community, and productivity. Ecosphere. 7: e01224. http://treesearch.fs.fed.us/pubs/52237

Krankina, O.N., Harmon, M.E., Schnekenburger, F., Sierra, C.A. 2012. Carbon balance on federal forest lands of Western Oregon and Washington: The impact of the Northwest Forest Plan. Forest Ecology and Management. 286: 171-182. 
Loreno, S., Fried, J.S., Yost, A. 2015. Applying management modeling to assess the feasibility of accelerating landscape restoration on federal forests in Eastern Oregon. In: Stanton, S. M., Christensen, G.A. (Eds). 2015. Pushing boundaries: new directions in inventory techniques and applications: Forest Inventory and Analysis (FIA) symposium 2015. 2015 December 8-10; Portland, Oregon. Gen. Tech. Rep. PNW-GTR-931. Portland, OR: U.S. Department of Agriculture, Forest Service, Pacific Northwest Research Station: 260-265. https://www.fs.usda.gov/treesearch/pubs/50355

Morton, J.M., Bowser, M., Berg, E., Magness, D., Eskelin, T. 2009. Long Term Ecological Monitoring Program on the Kenai National Wildlife Refuge, Alaska: An FIA adjunct inventory. In: McWilliams, W., Moisen, G., Czaplewski, R. (eds). Forest Inventory and Analysis (FIA) Symposium 2008; October 21-23, 2008; Park City, UT. Proc. RMRS-P-56CD. Fort Collins, CO: U.S. Department of Agriculture, Forest Service, Rocky Mountain Research Station. 17 p. https://www.fs.usda.gov/treesearch/pubs/33332

Ohmann, J.L., Gregory, M.J. 2002. Predictive mapping of forest composition and structure with direct gradient analysis and nearest neighbor imputation in coastal Oregon, USA. Canadian Journal of Forest Research. 32: 725-741. http://www.treesearch.fs.fed.us/pubs/ 4747

Schulz, B.K., Bechtold, W.A., Zarnoch, S.J. 2009. Sampling and estimation procedures for the vegetation diversity and structure indicator. General Technical Report PNW-GTR-781. Portland, OR: USDA Forest Service Pacific Northwest Research Station. 53 p. http://www. treesearch.fs.fed.us/pubs/32154

Scott, C.T.; Bechtold, W.A.; Reams, G.A.; Smith, W.D.; Westfall, J.A.; Hansen, M.H.; Moisen, G.G. 2005. Sample-based estimators used by the forest inventory and analysis national information management system. In: Bechtold, W.A.; Patterson, P.L. (Eds.), The enhanced Forest Inventory and Analysis Program-national sampling design and estimation procedures. USDA Forest Service, Southern Research Station, Asheville, NC, pp. 43-67. http: //www.treesearch.fs.fed.us/pubs/20371

Westfall, J.A., Patterson, P.L., Coulston, J.W. 2011. Post-stratified estimation: with-in strata and total sample size recommendations. Canadian Journal of Forest Research. 41: 11301139. https://www.fs.usda.gov/treesearch/pubs/37876

Woudenberg, S.W., Conkling, B.L., O’Connell, B.M., LaPoint, E.B., Turner, J.A., Waddell, K.L. 2010. The Forest Inventory and Analysis Database: Database description and users manual version 4.0 for Phase 2. General Technical Report RMRS-GTR-245. Fort Collins, CO: U.S. Department of Agriculture, Forest Service, Rocky Mountain Research Station. 339 p. http://www.treesearch.fs.fed.us/pubs/37446

Zheng, D.L., Heath, L.S., Ducey, M.J., Smith, J.E. 2011. Carbon changes in conterminous US forests associated with growth and major disturbances: 1992-2001. Environmental Research Letters. 6: 0140212. 